\title{
Virtual Traffic Police
}

\author{
R. Rakshitha ${ }^{1 *}$, M. J. Sudhamani ${ }^{2}$ \\ ${ }^{l}$ M.Tech. Student, Dept. of Computer Science and Engineering, RNS Institute of Technology, Bengaluru, India \\ ${ }^{2}$ Assistant Professor, Department of Computer Science and Engineering, RNS Institute of Technology, \\ Bengaluru, India \\ *Corresponding author: gowdarakshitha20@gmail.com
}

\begin{abstract}
In the new advancing world, traffic rule infringement has become a focal issue for larger part of the creating nations. The quantities of vehicles are expanding quickly just as the quantities of traffic rule infringement are expanding exponentially. Overseeing traffic rule infringement has consistently been a lethal and trading off undertaking. Despite the fact that the procedure of traffic the executives has gotten robotized, it's an extremely testing issue, because of the decent variety of plate designs, various scales, revolutions and nonuniform brightening conditions during picture obtaining. The vital target of this undertaking is to control the traffic rule infringement precisely and cost adequately. The undertaking presents Automatic Number Plate Recognition (ANPR) strategies and other picture control methods for plate confinement and character acknowledgment which makes it quicker and simpler to recognize the number plates. In the wake of perceiving the vehicle number from number plate the SMS based module is utilized to advise the vehicle proprietors about their traffic rule infringement. An extra SMS is sent to Regional Transport Office (RTO) for following the report status and furthermore to the proprietor of vehicles to advise about the standard infringement.
\end{abstract}

Keywords: CNN algorithm, Haar cascade, Number plate recognition, OCR method, Twilio, Yolo algorithm.

\section{Introduction}

In the previous hardly any decades, noteworthy endeavors in the field of moving article recognition and following have been done to make following applications dependable, powerful and proficient: video observation, mechanical technology, verification framework, media creation, natural research and so on. Be that as it may, there are numerous difficulties which produce leaps in the improvement of these applications. These difficulties may incorporate light change, dynamic foundation, cover, impediment, shadow and so forth. The quantity of mishaps on the streets is because of the standard infringement. To stay away from such petty criminal offenses, traffic police must be accessible out and has to patiently make sure if some vehicle is disregarding this standard.

A specific computerized arrangements were created to dispose of the infringement; anyway every one of them had certain confinements. For instance, the video catching cameras wiped out need of a position to be available to check rule infringement. Be that as it may, entire put away video must be checked physically for the standard infringement situation. In the proposed framework, an answer for signal breaking infringement is given. The framework incorporates a mechanized framework by utilizing IR sensor, camera and number plate acknowledgment application. In the framework IR sensor will be set close to zebra crossing line. In the event that any vehicle crosses the zebra line, the work area application will be started and will catch number plate picture.

Number plate acknowledgment application by utilizing picture handling calculation will perceive number plate and SMS will be sent to the guilty party if there should arise an occurrence of rule infringement scenario. Solid in all the nations there are driving guidelines accessible for individuals to drive cautiously by being straightforward and regarding.

At the point when these standards are broken it is characterized as a street infringement. There road encroachment that happens in ordinary busy time gridlock. These road shows are applied totally for the spots where by far most of the disasters happen. This structure is named as "VIRTUAL- POLICE". This structure is realized to encourage the work to the police using a UI. Picture getting ready development is used to recognize the ways, vehicles and will perceive vehicles who disturb standards. The gathering has realized the structure with the ultrasonic sensors in order to system to get conditions to recognize when an encroachment occur. It will be less difficult for the police to get who exceed laws of the traffic and for the cops who acknowledge quiet cash and dishonest people will in like manner be lessened.

A picture of the infringement with the area, time, date and a picture of the vehicle to the closest police officer's gadgets by determining gadgets around the present area that infringement occurred.

For instance, "When a vehicle speeds and on the off chance that it cuts the line on a traffic light when it is on red light proposed framework will recognize them and take photographs of the vehicle as confirmations as per the convention that is down and out and send a caution to the closest cops gadget" this examination is for the most part thought to be tied in with getting who defy the guidelines. This framework covers not many parts, for example, to recognize vehicles who overwhelm in wrong places, vehicles that velocities and traffic light convention infringement. This framework will be increasingly dependable and vehicle discovery and convention 
distinguishing proof will be accurate deviation of the centroids of the masses to perceive the event of the strange occasions. Robotization in everyday life has picked up significance as of late. The quantity of mishaps on the streets is because of the standard infringement.

A specific computerized arrangements were created to dispense with the infringement; anyway every one of them had certain impediments. For instance, the video catching cameras disposed of need of a power to be available to check rule infringement. Notwithstanding, entire put away video must be checked physically for the standard infringement scenario [4]. In this proposed framework, an answer for signal breaking infringement is given. The framework incorporates a robotized framework by utilizing IR sensor, camera and number plate acknowledgment application [3]. In this framework IR sensor will be set close to zebra crossing line. In the event that any vehicle crosses the zebra line, the work area application will be started and will catch number plate picture. Number plate acknowledgment application by utilizing picture preparing calculation will perceive number plate and SMS will be sent to the guilty party if there should arise an occurrence of rule infringement scenario [2].

The customary OCR based methodology for number plate acknowledgment doesn't work for the varieties in painting style of the number plates. In this paper creators have introduced a picture recovery based strategy to perceive the vehicle number plate caught utilizing an advanced cell to encourage the Car the board arrangement of a Smart office premise. In the proposed strategy an advanced mobile phone is utilized to catch the pictures and concentrate highlights of the vehicle number plate. These highlights are coordinated against predefined set of same vehicle number plate pictures in the database. The character pictures are coordinated in a proficient way to make it an ongoing arrangement.

These days there are some financially open applications like Google Goggles that see pictures and substance on the propelled cell, got by the PDA's camera. Such applications face the going with limitations and challenges like: (I) need of Internet Connection, (ii) essential to remove particular characters on embedded convenient stage which have objectives concerning both memory and processor, (iii) the closeness of specular reflection makes the affirmation task progressively irksome, (iv) pictures got are generally in the night, so the photos could be darkened and have low multifaceted nature (v) content Localization from the Background of the image got is a test. So we have endeavored to use an image recuperation based system for seeing the number plate. In that work the makers have shown a method to faultlessly interface physical and propelled world using paper records anyway a comparative technique can't be passed on in our anxiety.

As for vehicle wellbeing, India meets just two out of the seven vehicle security measures by the World Health Organization (WHO). Bikes represent $25 \%$ of complete street crash passings.

\section{Problem Statement}

The errand expects to give total prosperity to bike riders. Starting late head defenders have been made compulsory, yet simultaneously people drive without tops. Bengaluru has approx. 50 lakh bicycle riders, which joins 500-600 accidents reliably out of which 300-400 are deadly. Bengaluru positions first in the city with respect to bicycles riders. Over the latest couple of years, there has been fast augmentation in number of road setbacks. On account of rise in road disasters, it has now gotten essential to make a structure to confine spontaneous passings. To keep a tab on the heads a couple of charge assortment counters use a structure using fiber optic sensors to thus organize a vehicle far out and tally the results with the manual sections. In any case this structure is exorbitant tangled and requires high upkeep. We intend to consider the various structures that can be used to replace such a system with a more affordable and capable other alternative.

Existing system:

- Embedded System

- Traffic detection system using Android

- RFID Technology is used for Vehicles to control speed limit.

- Vehicle track

Limitation of existing system:

The current frameworks endured numerous issues like just the traffic status specifically region and following just vehicles which has RFID labels.

\section{A. Proposed system}

Tag acknowledgment (LPR) is one type of ITS (Intelligent Transport System) innovation that not just perceives and checks the quantity of vehicles yet in addition separates them. For certain applications, for example, electronic cost assortment and red-light infringement implementation, LPR records tags alphanumerically so the vehicle proprietor can be surveyed the suitable measure of fine. In others cases, similar to business vehicle activities or secure-get to control, a vehicle's tag is thought about against a database of adequate ones to decide if a truck can sidestep a checkpoint or a vehicle can enter a gated network or parking garage. A tag is the exceptional recognizable proof of a vehicle. The essential issues in real time tag acknowledgment are the precision and the acknowledgment speed. Tag Recognition (LPR) has been applied in various applications, for example, naturally distinguishing vehicles in parking garages, get to control in a limited zone and recognizing and confirming taken vehicles. Nature of calculations utilized in a tag identifier decides the speed and exactness of the tag location. Before, various methods have been proposed for finding the plate through visual picture preparing.

A video is taken from a camera, and afterward each casing of the video is prepared as the picture. In this stage the tag district from the given picture is found and disengaged. Nature of the 
picture has a significant influence henceforth before this stage pre-preparing of the picture is essential. So first each edge preprepared by linearization, commotion decrease and edge identification. At that point, the tag is situated by various picture preparing method.

Advantages of proposed system:

- This System is used to detect and read number plates automatically $24 / 7$ in real time.

- Installed model will help travelers to obey traffic rules.

- Can operate simultaneously in multiple lanes.

- Model detects and recognizes different dimensions, contrast, colors number plates with variety of character font and style.

\section{Literature Survey}

An altered number plate attestation Automatic Number Plate Recognition framework is a key point in active time gridlock hinder. This will help with constraining the assorted kind of encroachment all over town. Moved systems for following and perceiving taken, unapproved vehicles rely upon robotized number plate affirmation development. This current paper's fundamental targets are to audit different strategies and propose our own calculation. A short audit is performed on the different techniques for number plate acknowledgment calculations. Further clarifications of the proposed calculation are delineated in graphical structures to show how the calculation functions. This paper finished up with tests and assessment results [1]. Optical Character Recognition framework (OCR) can be utilized in savvy transportation frameworks for tag identification. Notwithstanding, most occasions the frameworks can't work with loud and defective pictures. In this work, a strong FPGA based OCR framework has been structured and tried with flawed and loud tag pictures. The OCR framework depends on a feedforward neural systems, which utilizes a proficient and exact neuron. The neuron move work depends on a guess of the Hyperbolic Tangent Activation Function. The system parameters were improved and afterward tried with boisterous pictures of tags numbers. The system had the option to keep up a 98:2\% exactness in perceiving the characters regardless of the picture blemishes [2]. Propelled Driving Assistant Systems, savvy and self-sufficient vehicles are promising answers for improve street security, traffic issues and travelers' solace. Such applications require propelled PC vision calculations that demand powerful PCs with rapid preparing capacities. Keeping keen vehicles out and about until its goal, at times, stays an incredible test, especially when driving at high speeds. The primary guideline task is strong route, which is regularly founded on framework vision to gain RGB pictures of the street for further developed preparing. The subsequent undertaking is the vehicle's dynamic controller as indicated by its position, speed and bearing. This paper presents a precise and proficient street limits and painted lines' location calculation for smart and self-governing vehicle. It consolidates
Hough Transform to introduce the calculation at each time required, and Canny edges' indicator, least-square technique and Kalman channel to limit the versatile locale of intrigue, redict the future street limits' area and lines parameters. The situations are reenacted on the Pro- SiVIC test system gave by Civitec, which is a reasonable test system of vehicles' elements, street foundations, and sensors practices, and OPAL-RT item devoted for continuous preparing and equal registering [3]. The utilization of vehicles throughout our life is expanding exponentially step by step and as expanding vehicles are damaging the traffic rules, robbery of vehicles, entering in confined territories, high number of mishaps lead to increment in the crime percentages straightly. For any vehicle to be perceived, vehicle tag identification will assume a significant huge job in this dynamic world. For discovering vehicles regularly utilized in field of security and wellbeing framework, LPDR assumes a noteworthy job and we have to perceive vehicles enrollment number at a specific separation [4]. The paper has four significant strides as follows: Preprocessing of caught picture, Extracting permit number plate district, Segmentation and Character Recognition of license plate. In pre-preparing the ideal vehicle image is taken through the computerized camera, brilliance of picture is balanced, clamor expulsion utilizing channels and picture is changed over to dark scale. Exactions of tag district comprise of finding the edges in the picture where definite area of licenses plate is found and yield it into rectangular edge. Division assumes an essential job in vehicle licenses plate acknowledgment; the decipherability of character acknowledgment totally depends on the division done. The methodology which we have utilized is basic however suitable. First we sectioned all characters in the picture (LP) utilizing Bounding box strategy. At last, acknowledgment of each character is finished. The layout coordinating technique is utilized for acknowledgment each character in the vehicle tag [5].

\section{A. System architecture}

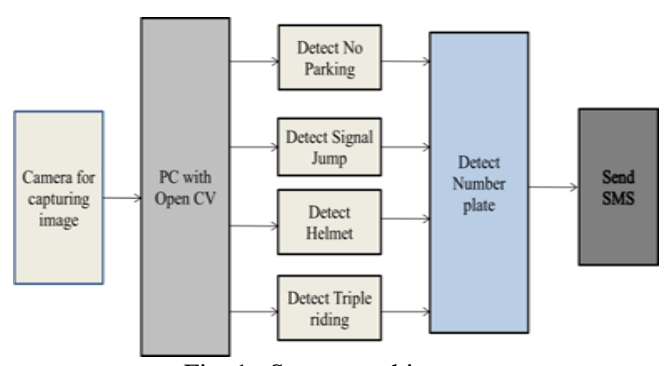

Fig. 1. System architecture

The proposed framework figure 1, will get the picture of the vehicles voyaging and minds the slim chance that any vehicle hurt the traffic rules, in the event that point of fact, by then the raspberry pi module will take starting and recognize the number plate of specific vehicle. Modified number-plate attestation is a progression that utilizes optical character certification on pictures to analyze vehicle enrollment plates to make vehicle locale information. It can utilize existing shut circuit TV, street 
rule need cameras, or cameras explicitly proposed for the undertaking. ANPR is utilized by police controls far and wide for law need purposes, including to check if a vehicle is chosen or endorsed. It is in addition utilized for electronic cost mix on pay-per-use streets and as a strategy for recording the progressions of traffic, for instance by interstates affiliations.

Modified number-plate affirmation can be used to store the photos got by the cameras similarly as the substance from the tag, with some configurable to store a photograph of the driver. ANPR advancement must consider plate arrangements here and there. The thing part of the structure runs on standard home PC outfit and can be related with different applications or databases. It first uses a development of picture control systems to see, standardize and overhaul the picture of the number plate, and a brief timeframe later optical character confirmation (OCR) to confine the alpha numerics of the tag. ANPR structures are generally sent in one of two key ways of thinking: one considers the whole philosophy to be performed at the way locale persistently, and the unmistakable transmits all the photographs from different approaches to a remote PC area and plays out the OCR procedure there at some later point in time. Precisely when done at the way site, the data got off the plate alphanumeric, date-time, way perceiving confirmation, and some other data required is done in around 250 milliseconds. This data can without an entirely momentous stretch be transmitted to a remote PC for extra preparing if essential, or put aside at the route for later recovery. In the other course of action, there are regularly colossal measures of PCs utilized in a server homestead to oversee high leftover loads, for example, those found in the London plug up charge experience. As frequently as conceivable in such structures, there is a fundamental to move pictures to the remote server, and this can require more prominent data move limit transmission media. The cameras used can be existing road rule usage or shut circuit TV cameras, similarly as compact units, which are ordinarily associated with vehicles. A couple of systems use infrared cameras to take an all the more away from of the plates.

Framework design is the strategy for describing a structure's engineering, parts, segments, interfaces, and data to satisfy the predefined preconditions. Design of systems could be deciphered as utilizing the hypothesis of structures to propel objects. The examination and procedures arranged in Article form into the most generally utilized strategies for the structure of PC systems. Along these lines, the arrangement of structures is the best approach to portray and assemble systems to meet the customer's characterized needs. The UML has become the standard language in object arranged examination and structure

The cameras used can be existing road rule usage or shut circuit TV cameras, similarly as compact units, which are ordinarily associated with vehicles. A couple of systems use infrared cameras to take an all the more away from of the plates.

Structure configuration is a material model that portrays the system's structure and conduct. This incorporates the casing pieces and the relationship clarifying how they cooperate to alter the general structure OCR (optical character confirmation) is the utilization of headway to see printed or deciphered substance characters inside computerized pictures of physical archives, for example, a filtered paper report. The essential arrangement of OCR consolidates looking at the substance of a record and causing an interpretation of the characters into code that to can be utilized for information arranging. OCR is from time to time in like way proposed as substance insistence. OCR systems are contained a blend of hardware and programming that is used to change over physical documents into machineclear substance. Gear, for instance, an optical scanner or specific circuit board is used to copy or read content while programming customarily handles the impelled getting ready. Programming can in like manner misuse man-made cognizance (AI) to complete additionally created procedures for sharp character affirmation (ICR), like distinctive tongues or styles of handwriting. The procedure of OCR is most normally used to transform printed copy legitimate or notable reports into PDFs. When put in this delicate duplicate, clients can alter, configuration and search the archive as though it was made with a word processor The initial step of OCR is utilizing a scanner to process the physical type of a report. When all pages are replicated, OCR programming changes over the archive into a two- shading, or high contrast, adaptation. The filtered in picture or bitmap is broke down for light and dim territories, where the dull regions are distinguished as characters that should be perceived and light zones are recognized as foundation. The dim regions are then prepared further to discover alphabetic letters or numeric digits. OCR projects can shift in their procedures, yet ordinarily include focusing on one character, word or square of content at once. Characters are then recognized utilizing one of two algorithms: Pattern acknowledgment OCR programs are taken care of instances of content in different text styles and organizations which are then used to think about, and perceive, characters in the examined report. Highlight discovery OCR programs apply rules with respect to the highlights of a particular letter or number to perceive characters in the checked report. Highlights could incorporate the quantity of calculated lines, crossed lines or bends in a character for correlation. For instance, the capital letter "A" might be put away as two slanting lines that meet with a level line over the center.

\section{B. Flowchart}

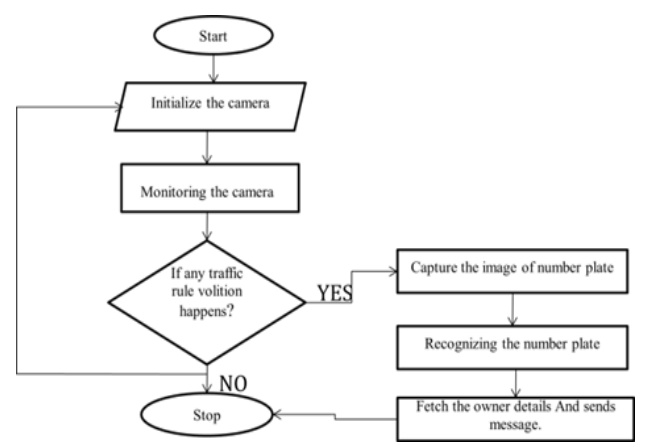

Fig. 2. Flow chart 


\section{IJRESM journals.resaim.com/ijresm | ISSN (Online): 2581-5792 | RESAIM Publishing}

The procedure of stream chart clarifies how precisely the virtual traffic police model works. Once in the wake of turning on the camera it begins working so that on the off chance that any vehicle violates any of the traffic rules, at that point the camera catches the picture of that specific vehicle this is the means by which the figure 2 works.

\section{Methodologies}

Lately, frames will be captured from video of rules violated vehicles and algorithm YOLO is being used to detect objects once after detecting object preprocessing will done to reduce the noise of the images. Algorithm CNN is used to classify images. Then openalpr is used which is an API use to detect the number plate by using method OCR. Once after recognizing the number plate of rule violated vehicle by using Twilio App a message will be sent to the respected owner of vehicles.

\section{Results and Discussions}

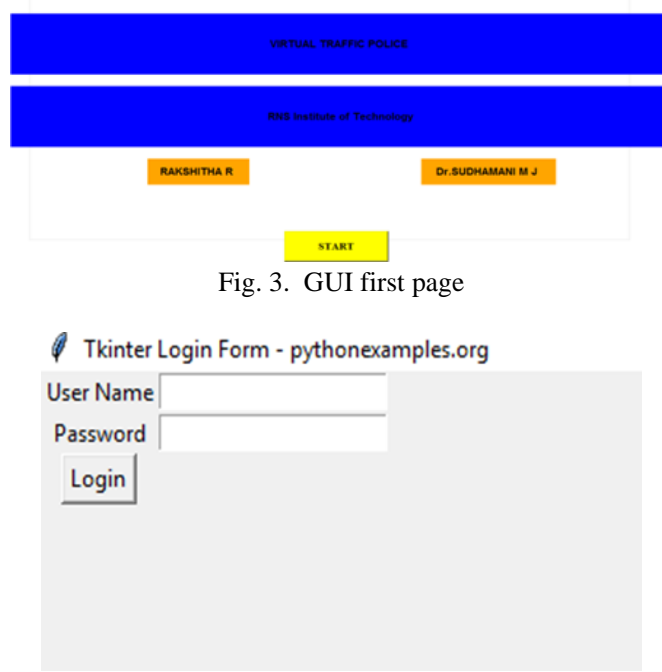

Fig. 4. Login page
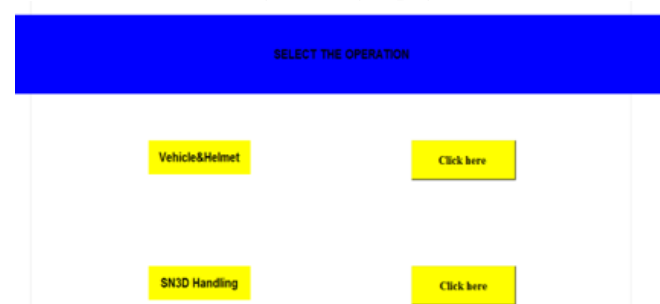

Fig. 5. Selection of operation to be performed

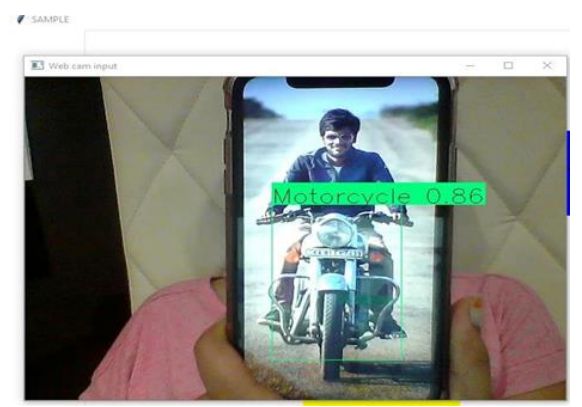

Fig. 6. Detection of motor cycle and helmet

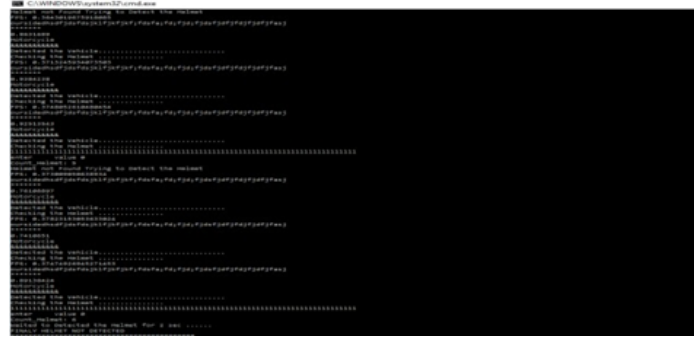

Fig. 7. Displaying of detected helmet, motor cycle and also helmet not found

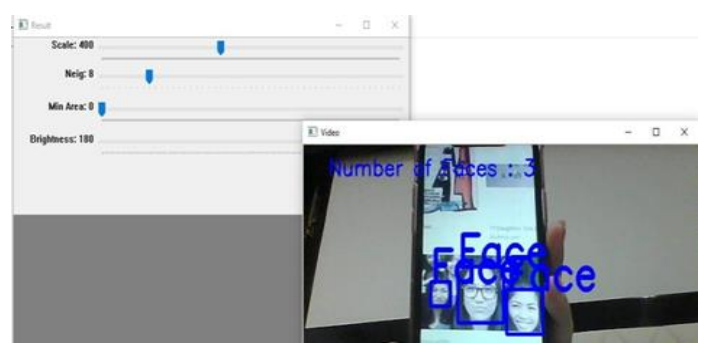

Fig. 8. Track bar and number of faces recognized

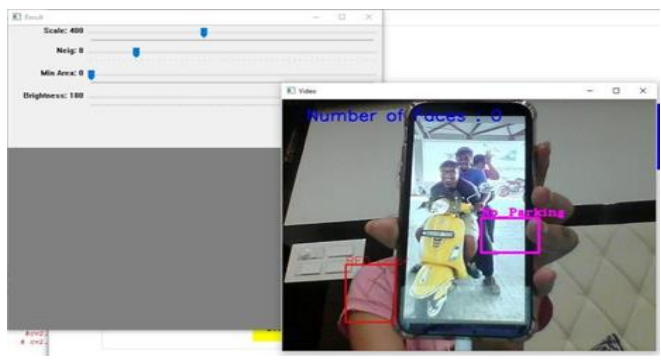

Fig. 9. Image of No parking, Red signal and triple riding on motor cycle

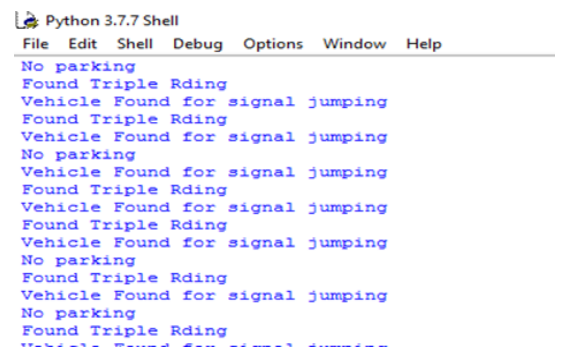

Fig. 10. Displaying of No parking, signal jump and triple riding

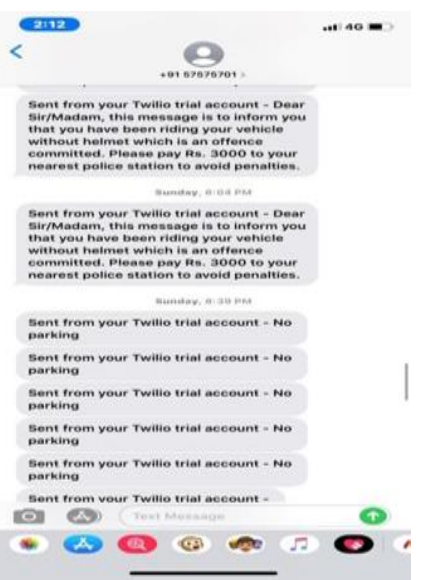

Fig. 11. Message received for rules violation 


\section{Conclusion}

The proposed framework is a replacement to human traffic cop. The strategies for traffic observation have been introduced and the work on movement identification, tag extraction and character acknowledgment is done. The proposed framework catches the picture in rush hour gridlock signal extraction and character acknowledgment of tag will be considered during rule infringement. Order of the traffic rule infringement will be done and the message will be send to the proprietor of the vehicle to illuminate them about the sort regarding rule abused and furthermore about the fine that must be paid.

\section{Future Enhancements}

The improvement of this model prompts the examination of new regions of robotization of traffic checking. The more precise component plan and increasingly hearty discovery strategy investigate will be executed in future. Shrewd bicycle can likewise be proposed which thus neglects to begin without wearing head protector. We can stretch out this framework to discover the quantity of traffic wrongdoers in a region.

\section{References}

[1] A. Ushma et al., "Object detection in image processing using edge detection techniques", IOSR journal of engineering, vol. 4, no. 3, pp. 1013, March 2014.

[2] D. Schrank, T. Lomax and S. Turner, "TTl's 2010 urban mobility report", tech. rep. Texas Transportation Institute, The Texas A\&M University System, December 2010.
[3] S. Krau, Microscopic Modeling of Traffic Flow: Investigation of Collision Free Vehicle Dynamics. Ph.D. thesis Mathematisches Institut Universität zu Köln, April 1998.

[4] M. Schnhof and D. Helbing, "Empirical features of congested traffic states and their implications fortraffic modeling", Transportation Science, vol. 41, pp. 135-166, May 2007

[5] C. Faloutsos, K. S. McCurley and A. Tomkins, "Fast discovery of connection subgraphs", KDD '04 Proceedings of the tenth ACM SIGKDD international conference on Knowledge discovery and data mining, August 2004

[6] H. Shang and X. Jin, "Indexing and mining of graph database based on interconnected subgraph", Intelligent Data Engineering and Automated Learning - IDEAL, vol. 4224, pp. 986-994, September 2006.

[7] J. L. Wilder, A. Milenkovic and E. Jovanov, "Smart wireless vehicle detection system", System Theory. 40th Southeastern Symposium, pp. 159-163, March 2008

[8] O. Jahn, R. H. Mhring, A. S. Schulz and N. E. S. Moses, "System-optimal routing of traffic flows with user constraints in networks with congestion", Operations Research.

[9] K. Collins and G.-M. Muntean, "A vehicle route management solution enabled by wireless vehicular networks", INFOCOM Workshops IEEE, pp. 1-6, April 2008.

[10] R. van Katwijk, P. van Koningsbruggen, B. D. Schutter and J. Hellendoorn, "Test bed for multiagent control systems in road traffic management", Transportation Research Record: Journal of the Transportation Research Board, vol. 1910, pp. 108-115, January 2005.

[11] J. Z. Hernandez, S. Ossowski and A. Garcia- Serrano, "Multiagent architectures for intelligent traffic management systems", Transportation Research Part C: Emerging Technologies, vol. 10, pp. 473- 506, November 2002.

[12] T. Yamashita, K. Izumi and K. Kurumatani, "Effective information sharing based on mass user support for reduction of traffic congestion", The Proceedings of International Conference of Complex Systems, 2004. 